\title{
Article
}

\section{One Source, Two Source(s): Ribs and Tethers}

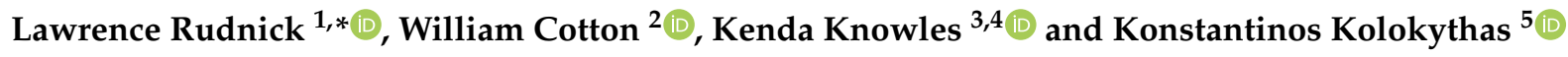 \\ 1 Minnesota Institute for Astrophysics, University of Minnesota, 116 Church St SE, \\ Minneapolis, MN 55455, USA \\ 2 National Radio Astronomy Observatory, Charlottesville, VA 22903, USA; bcotton@nrao.edu \\ 3 Department of Physics and Electronics, Rhodes University, P.O. Box 94, Makhanda 6140, South Africa; \\ kendaknowles.astro@gmail.com \\ 4 South African Radio Astronomy Observatory, 2 Fir Street, Observatory, Cape Town 7405, South Africa \\ 5 Centre for Space Research, North-West University, Potchefstroom 2520, South Africa; k.kolok@nwu.ac.za \\ * Correspondence: larry@umn.edu; Tel.: +1-612-624-3396
}

\section{check for}

updates

Citation: Rudnick, L.; Cotton, W.; Knowles, K.; Kolokythas, K. One Source, Two Source(s): Ribs and Tethers. Galaxies 2021, 9, 81. https:// doi.org/10.3390/galaxies9040081

Academic Editor: Stefano Ettori

Received: 3 October 2021

Accepted: 18 October 2021

Published: 23 October 2021

Publisher's Note: MDPI stays neutral with regard to jurisdictional claims in published maps and institutional affiliations.

Copyright: (c) 2021 by the authors. Licensee MDPI, Basel, Switzerland. This article is an open access article distributed under the terms and conditions of the Creative Commons Attribution (CC BY) license (https:/ / creativecommons.org/licenses/by/ $4.0 /)$.

\begin{abstract}
We present the unique and challenging case of a radio galaxy in Abell 3266 observed as part of the MeerKAT Galaxy Cluster Legacy Survey. It has quasi-periodic bright patches along the tail which connect to never-before-seen thin transverse extensions, which we call "ribs", reaching up to $\sim 50 \mathrm{kpc}$ from the central axis of the tail. At a distance of $\sim 400 \mathrm{kpc}$ from the host (assuming the $z=0.0594$ redshift of Abell 3266) we found what appears to be a triple source with its own apparent host at a photometric redshift of 0.78. Mysteriously, the part of the tail far from the host and the triple are connected by a series of thin filaments, which we call "tethers". The far tail, tethers and triple also have similar spectra and Faraday rotation measures, suggesting that there is only one-quite complicated-source, with a serendipitous background AGN in the triple. We look at possible causes for the "rib" and "tether" structures, and the emerging phenomena of intracluster medium filaments associated with radio galaxies.
\end{abstract}

Keywords: radio galaxy; clusters of galaxies; radio jets

\section{Introduction}

The study of radio galaxies is entering a new phase, with the exciting capabilities of SKA precursors such as MeerKAT and ASKAP; low frequency imaging at high resolution with LOFAR; and the high sensitivity, resolution and broad frequency coverage of the Jansky VLA. We are finding not only new examples of astrophysics-e.g., how radio galaxies interact with their environments—but new phenomena that may require revision of our basic theoretical pictures of jet physics. Here, we present one such case, which invites us to revisit what we assume we know about jets and their tails.

The mysterious radio galaxy in question, which we hereinafter call MysTail, is a member of Abell 3266. Initial results on this source have been presented in Knowles et al. [1]. The cluster is part of the Horologium-Reticulumin supercluster, and is in the midst of a complex merger, with velocities spanning 14,000 to $22,000 \mathrm{~km} / \mathrm{s}$ over a radius of $1^{\circ}$, or $\sim 4 \mathrm{Mpc}$ at the central cluster redshift of 0.0594 Dehghan et al. [2]. The core region of the cluster, shown in Figures 1 and 2, has two major components separated along a NE to SW axis, with a number of other filaments and subclusters to the north. Dehghan et al. [2] suggested that the ongoing merger activity suggests a high level of turbulence in the intracluster medium (ICM). The location of MysTail relative to the cluster is shown in Figure 1. MysTail is elongated at the edge, and along the same axis, as the major velocity components in the cluster core. This structure was previously seen by Bernardi et al. [3] with the MeerKAT precursor, KAT-7, although they were unable to classify its nature. 


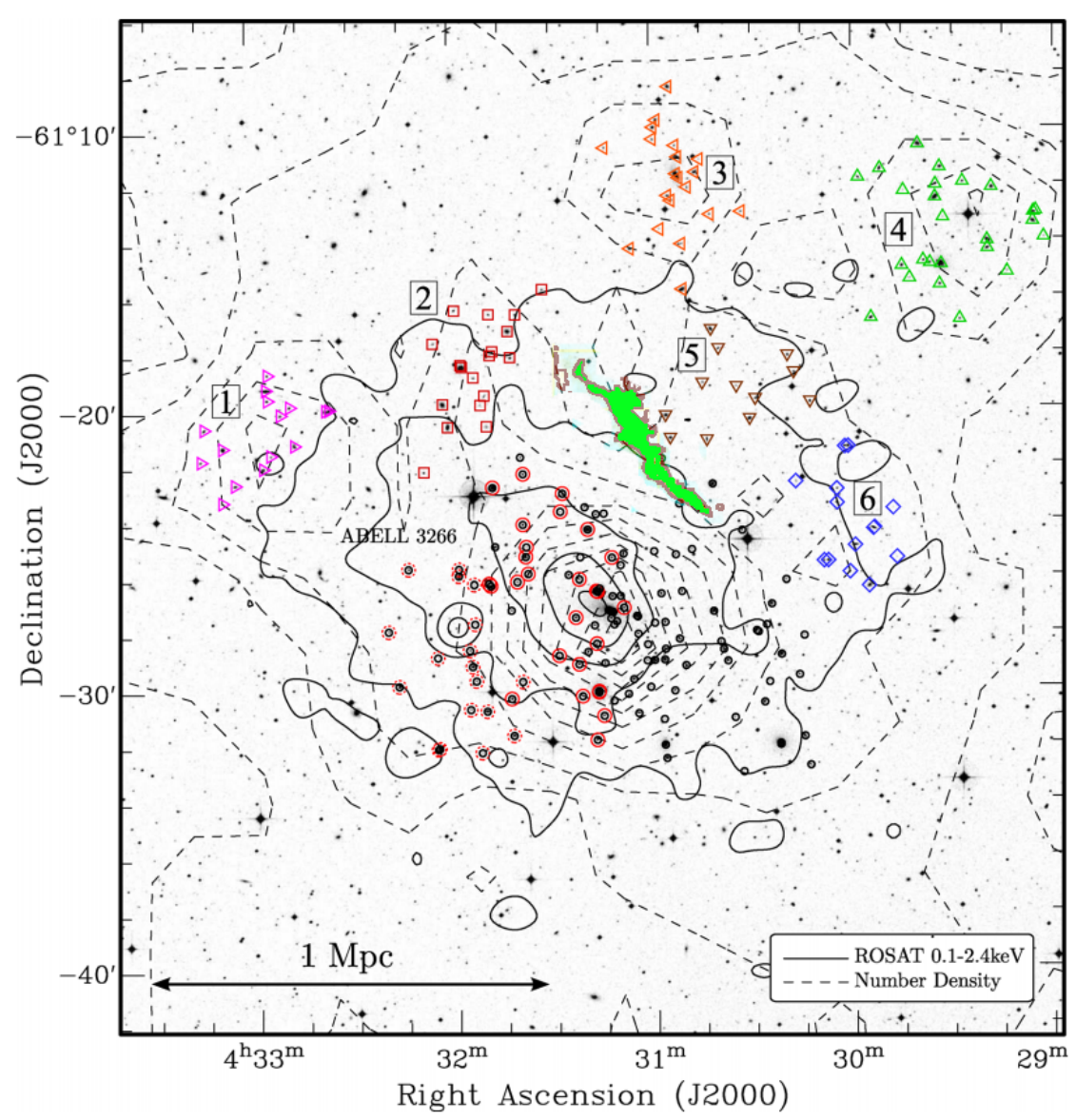

Figure 1. Distribution of optical galaxies in Abell 3266 from Dehghan et al. [2], (their Figure 7), showing the location and size of MysTail in light green.

The X-ray structure (Finoguenov et al. [4]) is elongated along the same NE-SW axis as the optical core, with unusual low entropy gas that they suggest has been stripped from an infalling subcluster. Recent eROSITA observations (Sanders et al. [5]) show an X-ray structure and pressure discontinuity further to the northwest, and other signs of the complex merger and possible AGN perturbations. An overlay of the radio image with the XMM-Newton X-ray image of the inner $\sim 15^{\prime}$ of the field is shown in Figure 2. Other interesting radio galaxies and radio relic structures are also present in the field, but are beyond this image and the scope of this work. 


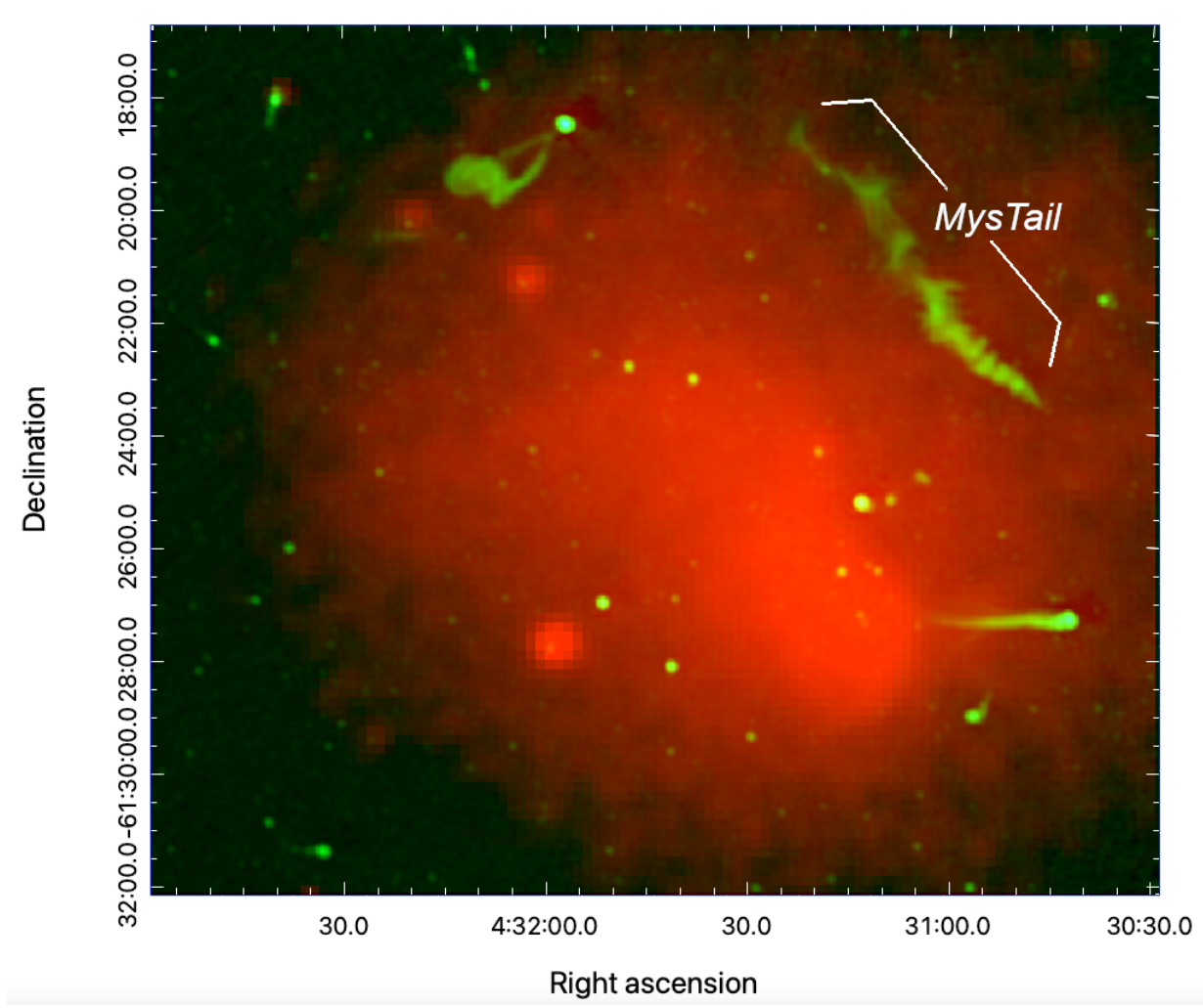

Figure 2. Radio map in green of the central $\sim 15$ arcmin of the cluster region, overlaid with a XMM-Newton 0.7-2.3 keV image, courtesy of Hiroki Akamatsu, in red.

\section{Observations and Overview}

The observations presented here were carried out as part of the MeerKAT Galaxy Cluster Legacy Survey (MGCLS; Knowles et al. [1]). Details of the observations and map production are given there, but we include a brief summary here.

The observations were made with the MeerKAT array on 3 March 2019 for $8 \mathrm{~h}$ using J0421-6223 as the gain calibrator and PMN J0408-6545 as the flux density/bandpass calibrator. The observed integration time was 8 seconds with $0.21 \mathrm{MHz}$ channels covering the nominal frequency range $900-1670 \mathrm{MHz}$. The primary antenna beam at mid-band is $67^{\prime} \mathrm{FWHM}$ and the resolution of the image is $7.1^{\prime \prime} \times 6.7^{\prime \prime}$ at position angle $31^{\circ}$. All four combinations of the two linear feeds were correlated.

Polarization calibration was as described in Knowles et al. [1] and used the noise signals injected at the beginning of each observing session and calibration parameters derived from external data sets. Imaging in Stokes $Q$ and $U$ used the same 5\% fractional bandwidths as were used for Stokes I.

After calibration, the data were averaged into $0.83 \mathrm{MHz}$ channels for imaging. At the location of MysTail, $350^{\prime \prime}$ from the field center, the RMS is $6 \mu \mathrm{Jy}$ beam $^{-1}$. The spectral index $\alpha$ (using the convention $S(v)=S_{0} v^{\alpha}$ ) was fit to each pixel using the sub-band images derived in the imaging after primary beam correction.

\section{Dissecting the Structure}

Figure 3 shows a close-up view of MysTail, indicating the structures discussed here and the proposed optical identifications. At present, we cannot determine whether we are looking at a single radio structure, or multiple structures either physically linked or seen only in projection. Another alternative is that MysTail could be a peripheral radio relic, because it follows the elongated X-rays in the cluster core. eROSITA studies show a parallel pressure jump structure, although it is much further out. The identification of MysTail as a tailed radio galaxy, however, is indicated by the existence of a radio core and coincident bright galaxy at one end (see right-most inset in Figure 3), coupled with 
the spectral behavior described below. The optical galaxy is WISEA J043045.39-612335.6 (6dFGS g0430454-612336), at a redshift of 0.0626, within the velocity distribution of the Abell 3266 core. The tail is resolved transversely $\left(\sim 20^{\prime \prime}\right.$ wide in the faint regions, with a $7.2^{\prime \prime}$ beam), but shows no signs of bifurcating into a twin-tailed structure. It is punctuated by quasi-periodic bright patches, and shows much more extended transverse structures, reaching over an arcminute in extent, which we label "ribs". At $2.1^{\prime}(\sim 150 \mathrm{kpc})$ from the core, the tail shows a sharp bend of $\sim 35^{\circ}$. It continues for $\sim 40^{\prime \prime}$ before bending again and getting dimmer. A bright patch appears $\sim 50^{\prime \prime}$ further downstream.

At the far (NE) region of the source is a structure which we label as the "triple", because of its similarity to a double-lobed radio galaxy with a core and connecting jets. Approximately half of the $0.64 \mathrm{mJy}_{\text {beam }}{ }^{-1}$ core brightness may come from the underlying jet. In addition to the cluster radio galaxies, there are approximately 15 compact radio sources with peak fluxes $>0.3 \mathrm{mJy}_{\text {beam }}{ }^{-1}$, within $1.2 \times 10^{5}$ square arcseconds; the chances of being located somewhere within the jet-like structures is thus less than a few percent. Coincident with the presumed core, we found a faint optical/IR galaxy (DES J043118.45611917.9 WISEA J043118.50-611918.2) with a photometric redshift of 0.78 (Zou et al. [6]). At this redshift, the 120-arcsec possible triple source would be $900 \mathrm{kpc}$ in length; if the triple were an extension of the tail, it would be $140 \mathrm{kpc}$ long. Between the bright patch at the end of the tail and the triple, we found three filamentary features, which we label as "tethers". The connection between all of these structures is unclear, hence the interest in this source.

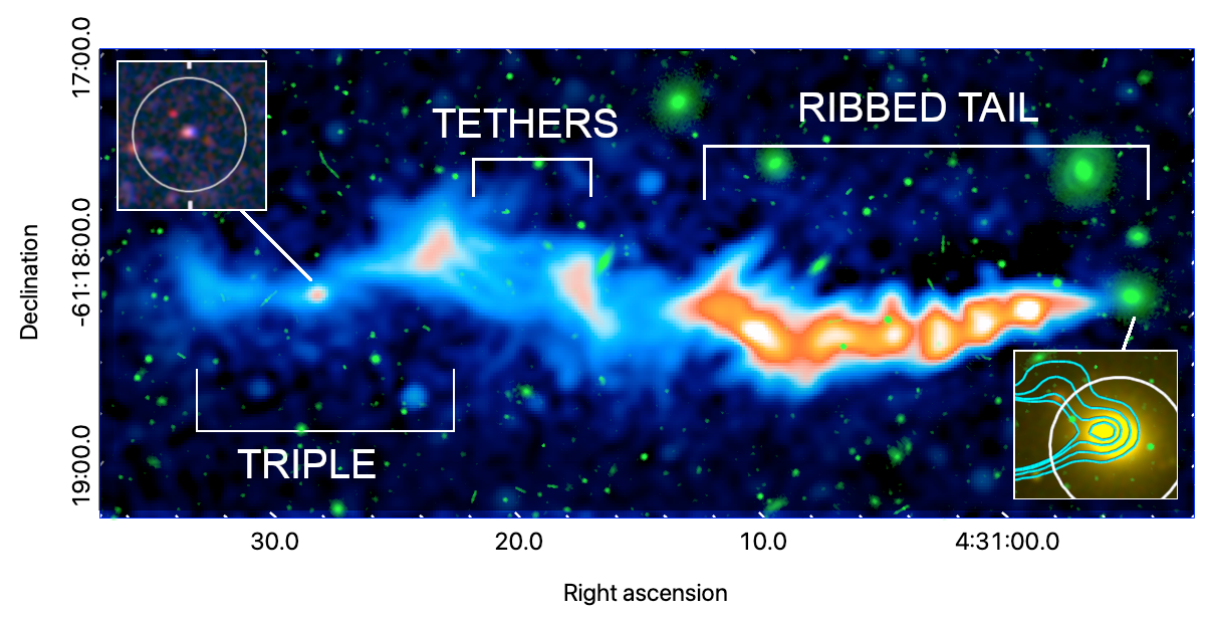

Figure 3. MGCLS $1285 \mathrm{MHz}$ radio map of MysTail with suggested host(s) and labeled features. The background green image and the insets show the optical field from the Dark Energy Survey. Radio contours on the inset indicate the presence of a core coincident with the suggested host.

\section{Source Diagnostics}

\subsection{Structure}

In order to look at the properties of the bright spots and ribs in more detail, we made transverse cuts at a number of locations along the tail and measured their peak brightnesses and Gaussian widths. Note that these width measurements are only approximate indicators, and include the broadening by the ribs. A more accurate width determination would require assumptions about the true underlying brightness profiles of the tail and ribs. The quasi-periodic brightening of the tail is seen in Figure 4. The characteristic separation between the bright spots is $\sim 20^{\prime \prime}$, comparable to the width of the tail. The tail widens over the first $200^{\prime \prime}$, past its initial bend. The ribs are shorter near the head, and appear mostly on one side, reaching $\sim 20 \mathrm{kpc}$ from the center line of the tail. The longest rib is seen at the end of the bend, and spans a total length of $\sim 100 \mathrm{kpc}$. The widths of the ribs range from about 4 to $7 \mathrm{kpc}$, after beam deconvolution. 

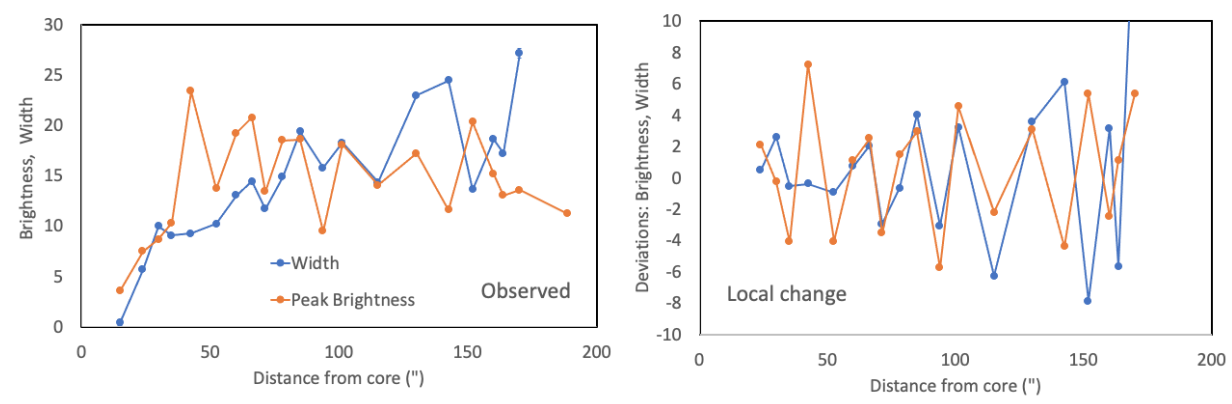

Figure 4. Gaussian fits of peak and deconvolved width to profiles across the tail. (Left) As observed, in units of $10^{-4} \mathrm{Jy}$ (peak) and arcsec (width); (Right) difference between each point and the average of its two neighbors, in units of (\% deviation)/10 (peak) and arcsec (width). Statistical error bars are approximately the sizes of the symbols; the true uncertainties are dominated by the non-Gaussianity of the profiles and the background subtractions.

In order to look more closely at the small-scale variations, we took out the large-scale trends by taking the difference between each peak and width and the average of the two neighboring measurements. The results are also shown in Figure 4. The key result here is the same as can be seen in the image; the ribs often occur at the bright patches in the tail, although this relationship gets confused around the bend, where the structures become more difficult to match up with one another.

\subsection{Spectral Index Variations}

The overall spectral index behavior, shown in Figure 5, is similar to what is seen in other tailed radio galaxies. The index is -0.6 near the core, steepening to $\sim-1.4$ at the bend in the tail. Beyond the second bend, there is a dramatic steepening with values ranging from $\sim-2$ to $\sim-3.4$ (except for the putative core of the triple, with $\alpha=-1.4$ ). We did several spot checks of the pixel-by-pixel spectral fits by integrating over larger areas in each of the frequency channel maps and refitting for the spectral index. The results were consistent. Note that the ribs follow the same overall spectral trends as the tail.

In addition, we found no evidence for spectral curvature, even when the spectra were steep, with changes in spectral index less than 0.5 between the top and bottom halves of the band. For comparison, an exponentially cut off spectrum with a lower frequency index of -0.6 would have steepened by $\sim 1.5$.

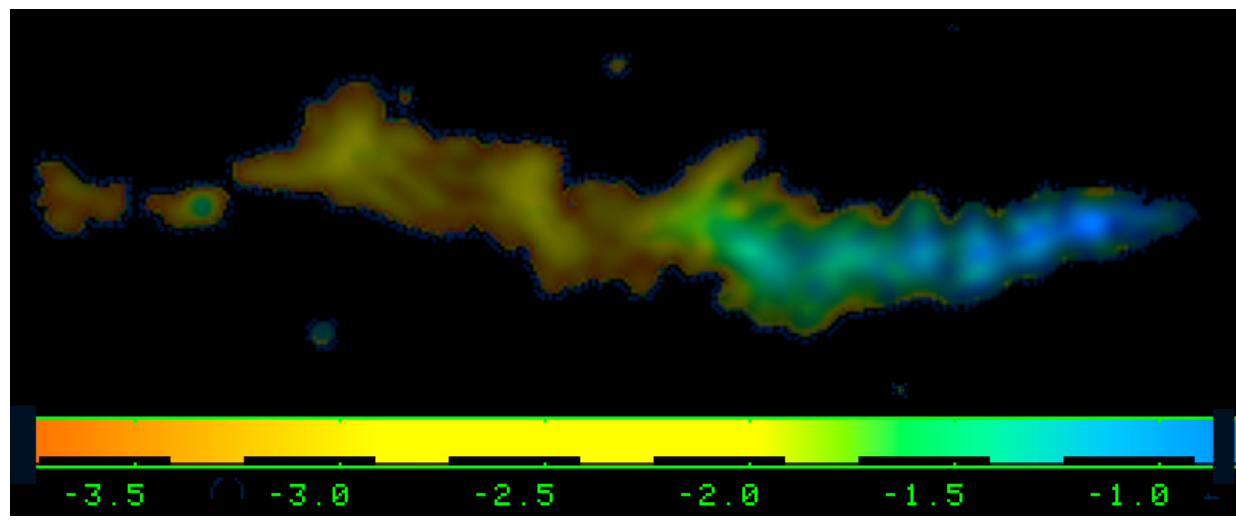

Figure 5. Spectral index color coding of the total intensity map.

A closer look at the spectral structure is shown in Figure 6. This image was made using "spectral tomography", which helps identify overlapping structures with different spectral indices (Katz-Stone and Rudnick [7]). There appear to be two parallel lines of slightly steeper spectrum material surrounding the ridge line of the tail, although the flatter spectrum emission extends transversely beyond those lines. This behavior can be seen in 
the profile plot, which shows a steepening away from the ridge line, with some modest flattening further out. Note that the total intensity cut blends all of these features together. The true underlying structure and spectral decomposition would require resolutions $<3^{\prime \prime}$; the results here show simply that the tail cross-section is not uniform.
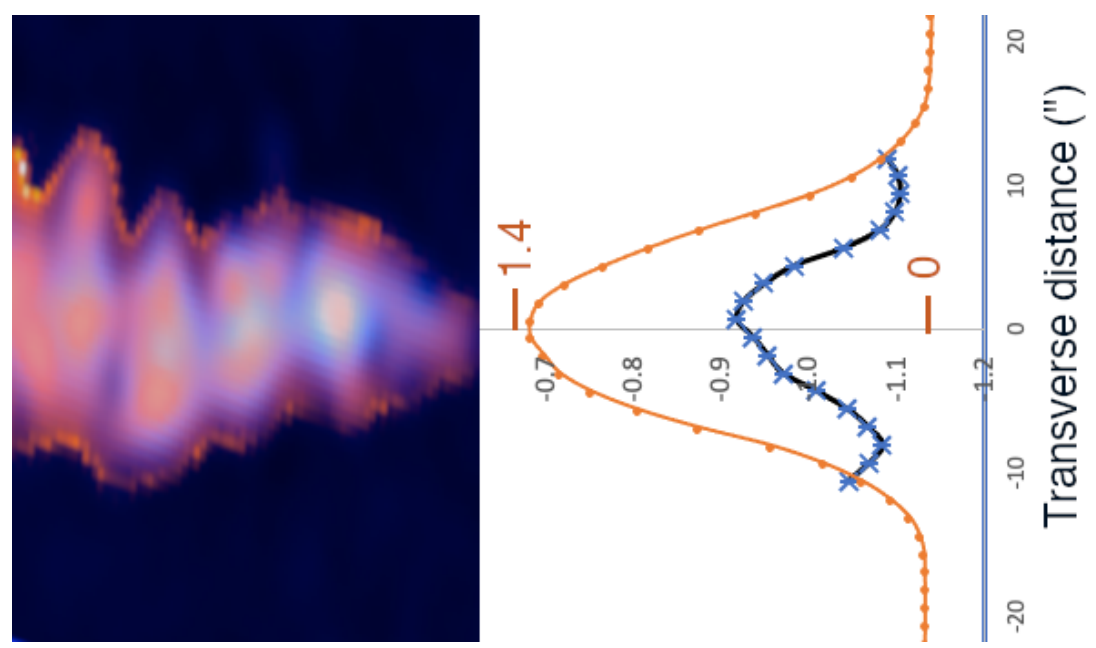

Figure 6. Closeup of beginning of tail to highlight spectral index structure, stretched vertically by a factor of 2. (Left): Spectral tomography images. Blue (orange) shows emission flatter than (steeper than) $\alpha=-0.63$. These cannot be used quantitatively; they simply illustrate the overall spectral structures. (Right): The profile cuts across the tail in total intensity (orange) and spectral index (blue); these were produced by taking the median value of the respective images along the $\sim 1^{\prime}$ portion of the tail shown here, at each distance from the tail central line.

\subsection{Tethers}

The tethers have an average brightness of $\sim 250 \mu \mathrm{Jy} /$ beam, comparable to the ribs, the fainter portions of the tail, and the triple. Their length is $\sim 45^{\prime \prime}$ (52 kpc, assuming they are associated with Abell 3266), and their widths are slightly resolved, with deconvolved values of $6-7^{\prime \prime}(7-8 \mathrm{kpc})$. Figure 7 shows a profile cut across the tethers, with a 3-Gaussian model. The blending of three slightly resolved Gaussians accounts for all the flux in the tether region, although it does not rule out a combination of multiple thinner structures and more diffuse emission that could become visible at higher resolution. A faint partial fourth tether is also visible at some locations. Cross-sectional cuts are also consistent there with three Gaussians, with the fourth tether replacing one of the two merged brighter tethers.
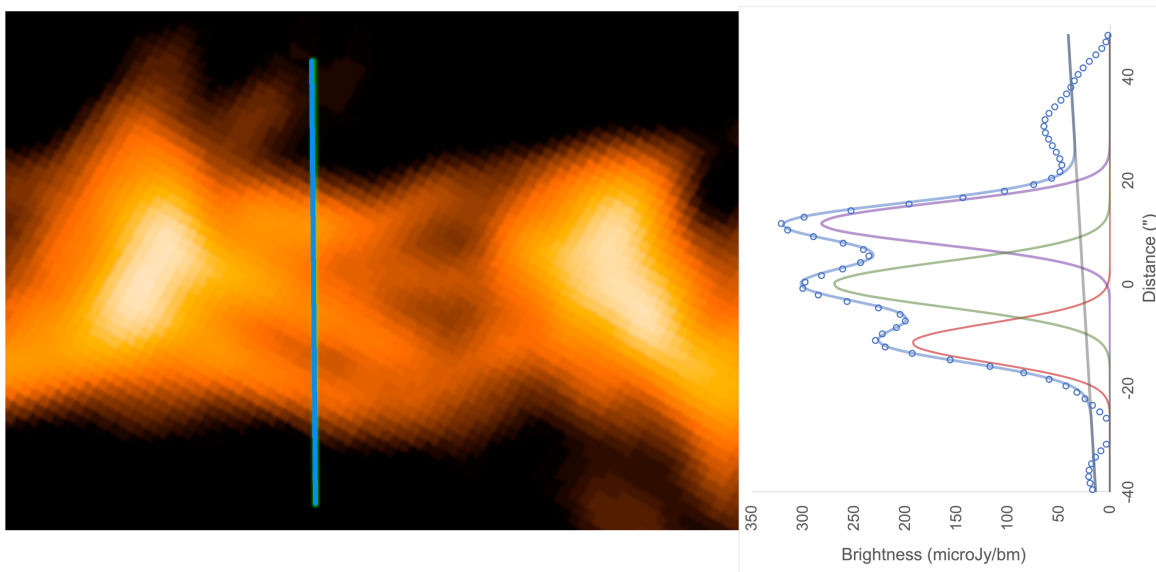

Figure 7. Closeup of the tethers region, with a profile cut showing that it can be characterized as three somewhat resolved Gaussians. 


\subsection{A First Look at Polarization}

The Faraday rotation in the field was derived from a Faraday synthesis of the $Q$ and $U$ image cubes. A direct search was made in each pixel between $+300 \mathrm{rad} \mathrm{m}^{-2}$ and $-300 \mathrm{rad} \mathrm{m}^{-2}$ with increments of $1 \mathrm{rad} \mathrm{m}^{-2}$. The value of rotation measure that produced the maximum unwrapped polarized emission was taken as the rotation measure of that pixel. The polarized intensity (at the peak in the Faraday spectrum) and the electric vector polarization angle corrected for Faraday rotation were then calculated.

The results are shown in Figure 8, where the signal:noise is high enough to determine a reliable RM. In the initial $125^{\prime \prime}$ section of the tail, prior to the bend, the average $\mathrm{RM}$ is $22 \mathrm{rad} \mathrm{m}^{-2}$, with an RMS scatter of $\sim 30 \mathrm{rad} \mathrm{m}^{-2}$. The polarization is seen to be patchy, as is the RM, with no obvious systematic orientation to the inferred magnetic field vectors. The fractional polarization, where it can be measured, averages $\sim 7 \%$.

There is a sharp transition in the RM values at the bend, with a mean $\sim 120 \mathrm{rad} \mathrm{m}^{-2}$, and scatter of $\sim 45 \mathrm{rad} \mathrm{m}^{-2}$. In general, the $\mathrm{RM}$ variations are on larger scales and the mean fractional polarization rose to $\sim 11 \%$. In addition, there is a compact region of high polarized flux at the bend, and a large jump in RM. These suggest some interaction with the surrounding thermal material. There is no significant change in the spectral index of $\sim-1.4$ at this position.

The RMs remain at similar high values in the region of the tethers, $\left(95 \pm 10 \mathrm{rad} \mathrm{m}^{-2}\right)$ and the triple $\left(75 \pm 35 \mathrm{rad} \mathrm{m}^{-2}\right)$, again with variations over larger scales than the initial section of the tail. The mean fractional polarization is higher still, $\sim 25 \%$. The magnetic fields in the large extended features, including the tethers, tend to align with the structures.

Significant changes in the polarization characteristics (RMs, field directions, and fractional polarizations) are thus seen to correlate with the overall source structure. Interactions with the surrounding medium are likely to play an important role in this behavior.
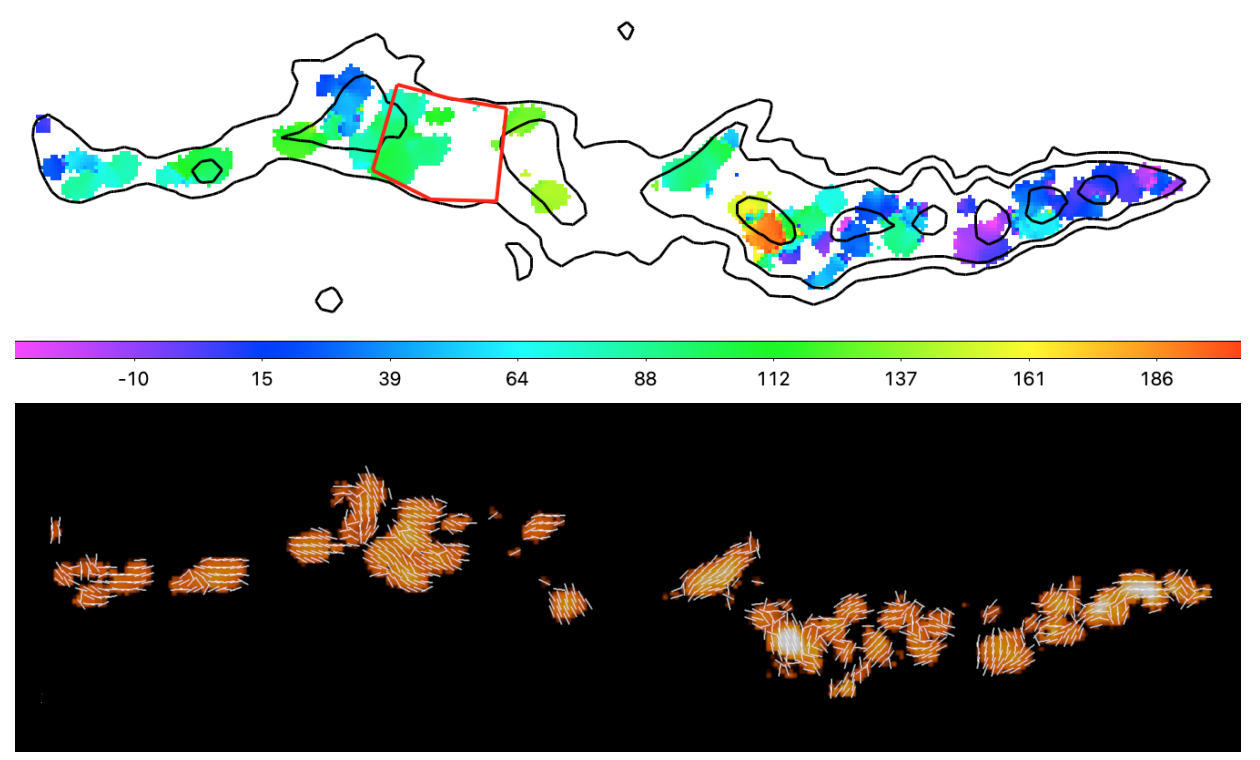

Figure 8. (Top) Rotation measure, with scale in $\mathrm{rad} \mathrm{m}^{-2}$ and total intensity contours spaced by factor of 4 , starting at $81 \mu \mathrm{Jy} \mathrm{beam}^{-1}$. The red polygon denotes the region of the tethers. (Bottom) A heatmap of polarized intensity ranging from 25 to $150 \mu \mathrm{Jy} / \mathrm{beam}$, superposed by inferred magnetic field direction vectors after correction for Faraday rotation.

\section{Discussion-What Are We Seeing?}

\subsection{Bright Patches and Ribs}

We briefly summarize the key features of these structures as follows:

- A radio tail with no visible bifurcation, with quasi-periodic brightness variations over its initial $200 \mathrm{kpc}$. 
- The brightness modulations are factors of $\sim 1.5$ with a separation between peaks in the order of two times the tail diameter.

- At the positions of the bright peaks, thin $(\sim 5 \mathrm{kpc})$ transverse "ribs" extend up to $\sim 100 \mathrm{kpc}$ in length across the tail.

- All of the structures have spectra which steepen with distance down the tail, with the ribs being typically locally steeper by about 0.2 than the core of the tail.

We note that quasi-periodic jet brightenings have been seen previously, including the spectacular example of PKS 0637-752 (Godfrey et al. [8]) with $>10$ knots spaced $\sim 7.6 \mathrm{kpc}$ apart (in projection). They interpreted these as being due to variations in jet output on timescales of $10^{3}-10^{5}$ years. Although no transverse structures were noted, deeper observations of this system are certainly warranted.

In general terms, there are three plausible ways in which these structures could arise: variations in the AGN jet output; instabilities in the flow (including possible interactions with an inhomogeneous external medium); coherent pre-existing external structures superposed on/interacting with the tail. We provide brief initial speculative thoughts on these possibilities.

Pulsed/varying jets? Temporal variability of flux and structure in jets is well-studied on pc-scales (Lister et al. [9]). At the other extreme, episodic jet activity has been proposed to explain much larger scale structures in double-double radio galaxies, and the curious multiple structures in a spiral radio galaxy (Sirothia et al. [10]). On kpc and larger scales, simulations with various jet duty-cycles have been used to study the feedback effects on the surrounding cluster medium or on galaxy formation (Birzan et al. [11]). Re-starting jet studies reveal rich underlying physics, such as the differences between jet injection into pre-existing channels from previous activity or jets that need to excavate a new path (Barniol-Duran et al. [12]). For MysTail, jet variability could be at play if the outbursts were highly overpressured and lead to transverse "ribs"; however, this has yet to be seen or predicted in simulations, so further work is needed. In addition, the spacing of the bright patches on similar scales to the jet diameter would be fortuitous.

Instabilities in the flow-recollimation shocks? Various instabilities naturally develop in flows, largely through interactions with the external medium (O'Neill et al. [13]). These can result in flapping-type motions, entrainment of external material, and the creation of vortex rings when shocks are encountered (Noltin et al. [14]). One interesting possibility is whether recollimation shocks are present; they can be created when jets over-expand and are then reconfined as they propagate into lower density environments (Sanders [15]). Mizuno et al. [16] has also explored the role of axial magnetic fields in pc-scale jets in strengthening recollimation shocks and the resulting compressions and rarefactions. Recollimation processes such as these might therefore be attractive possibilities for creating the bright knots, but the broadening of the jets at the bright spots and the development of "ribs" do not appear to be consistent with this process.

External (ICM) structures? Pre-existing density fluctuations, or especially, filamentary structures, in the ICM are attractive candidates for the ribs, and are discussed further below in the context of the "tethers". We also note that large, thin transverse features have recently been found in Centaurus A, and associated with bright knots in its Northern Middle Lobe (B. McKinley, private communication [17]). In that case, the strong mildly supersonic winds from the AGN appear to be interacting with clumps of X-ray emitting material, giving rise to these features. There is no indication or expectation that this particular mechanism would be applicable to MysTail.

\subsection{Tethers-Connecting One or Two Sources?}

To interpret the tethers, we must first determine whether the tail, which itself is clearly part of Abell 3266, is physically associated with the triple. Either way, we are faced with an unsettling coincidence. If these are one physical source, then the optical/radio "core" must be an accidental background source. We calculated a 3\% chance that such an object would be found somewhere along the apparent jet-like structures in the triple. If we also 
note that at the approximate central position of the core, the probabilities would go down by another factor of 5 .

At present, accepting this coincidence appears preferable to the coincidences we would have to accept if these are two separate sources, as seen in the projection. These include:

- That the tail and triple fall along the same line, and appear connected by thin filaments (the tethers).

- That the spectral index smoothly steepens from the tail, through the tethers, and from the west to the east through the triple. The very steep spectra in the triple would also be unusual for a random background source, although more typical for the faint regions of a tail.

- That the RM distribution in the triple is similar to that of the tethers and the post-bend regions of the tail, and finally;

- That the triple is parallel to, and appears to be located at/near, a drop in the cluster X-ray brightness, as shown in Figure 9; note that this drop is located interior to the pressure jump seen with eROSITA (Sanders et al. [5]).

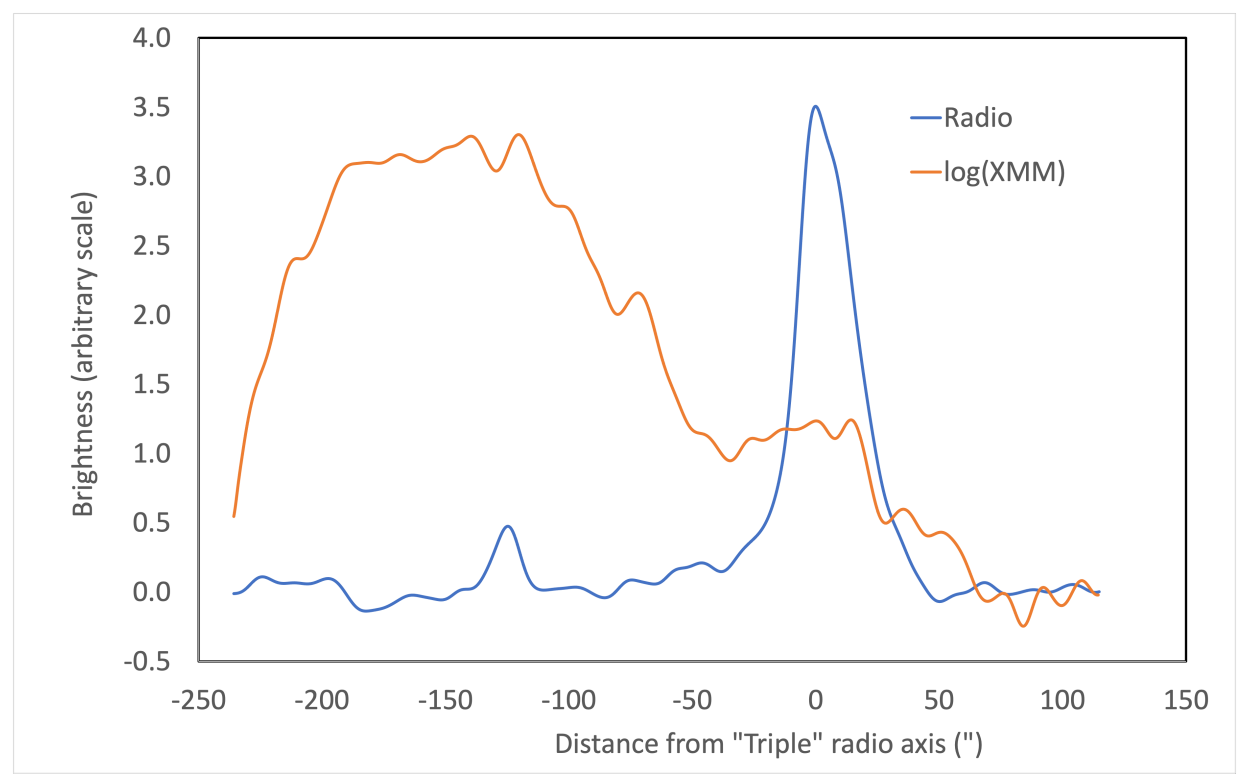

Figure 9. Brightness slices in radio and X-ray (from XMM) perpendicular to the "triple". These were produced by first convolving the images with a Gaussian of $125^{\prime \prime} \times 10^{\prime \prime}$ at an angle of $44 \mathrm{deg}$, along the triple structure. The brightness scales are arbitrary, and in log brightness for the X-rays. These plots show that the triple is located at/near a drop in X-ray brightness.

We therefore adopt, for the purposes of discussion, that we are looking at one source comprising the tail, the triple, and the connecting tethers. Other than the unusual nature of the ribs, as discussed above, the tail is similar to many other tailed radio galaxies that have been studied over the years. The triple structure, the somewhat isolated bright patch between the tethers, and the tail and the tethers themselves are quite unusual-but not unique. Similar features, e.g., are seen in IC711 (van Weeren et al. [18]), with patchy structures, multiple filamentary structures intersecting with the tail, possibly connecting to other sources in the host cluster Abell 1314, etc. (see Figure 10). The interactions of radio tails with the ICM are likely much richer than we have observed or modeled through simulations, up until now. 


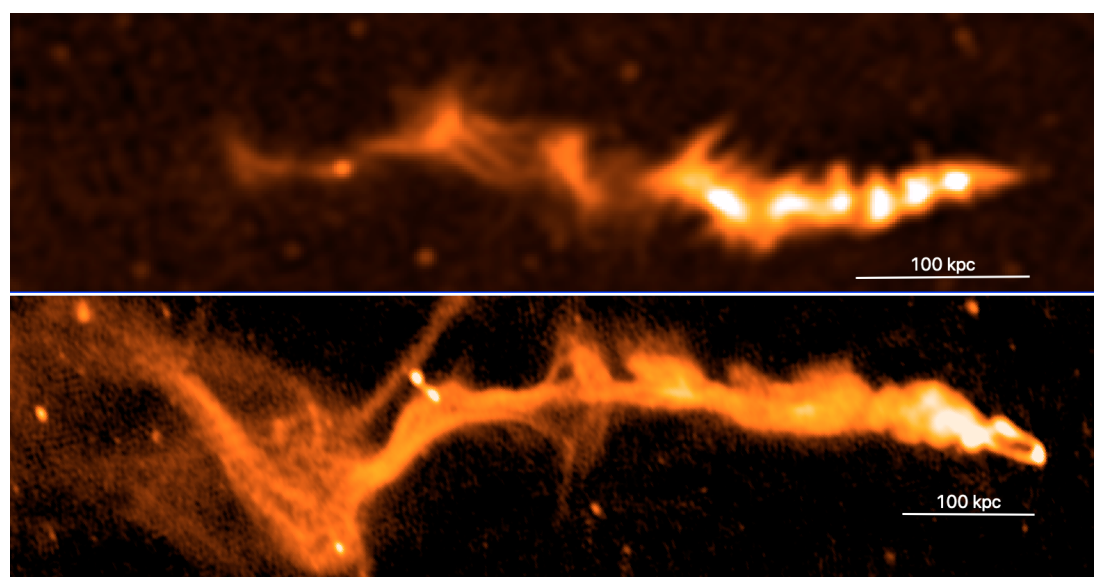

Figure 10. MeerKAT observations of MysTail, as presented here, and image produced using LOFAR observations of IC711, courtesy of van Weeren et al. [18], on similar scales, showing the emergence of heretofore unusual features associated with tailed radio galaxies.

Filamentary features associated with radio galaxies are becoming more common. An extensive network of filaments was found in Abell 194, also observed as part of the MGCLS Survey (Knowles et al. [1]), around the radio galaxies 3C40A,B. Ramatsoku et al. [19] showed multiple "collimated synchrotron threads" connecting the lobes of radio galaxy ESO 137-006. Condon et al. [20] described the "threads" and "ribbons" in IC4296, which they suggested are related to instabilities in the jets and relics of earlier jet activity, respectively. Even the well-studied radio galaxies 3C129, NGC 326, and IC711 have revealed strange filamentary features connected with their more conventional structures van Weeren et al. [18], Lane et al. [21], Hardcastle et al. [22].

Filamentation may thus occur naturally in the ICM, with the magnetized filaments becoming populated, and thus illuminated by relativistic particles when reconnection takes place with radio galaxy lobes. Similar processes may play out in our own galaxy, where massive winds in the galactic center interact with strong fields in the interstellar medium, leading to equally spaced structures called "synchrotron harps" by Thomas et al. [23]. Such structures could also be responsible for the "ribs" discussed above. If such filaments intersected with the tail, and magnetic field reconnection took place, it is possible that they would become illuminated by relativistic electrons from the tail. Diffusion times, spectral expectations, lengths, and widths - these are all aspects that would need study to make such a scenario plausible.

\subsection{The Tail-X-ray Connection}

Radio galaxies are clearly distorted by their interactions with the ICM, and in the dramatic case of encountering shocks, may be responsible for forming peripheral radio relics (e.g., Jones et al. [24]). Although there is no shock structure apparent at the location of MysTail, it does lie along the edge of the low-entropy bright central cluster emission, and its structure could be associated with flows along or pressure gradients across this edge. The spectral gradients along its length suggest that the relativistic electrons are still evolving passively as expected down a tail, but the unusual structures might indicate interactions with the ICM. The sharp bend in the tail at $\sim 150 \mathrm{kpc}$ from the core is accompanied by changes in the Faraday and magnetic field structure; these are likely signatures of a change in the physical and/or dynamical properties of the local ICM.

\section{Conclusions}

In the end, MysTail presents several mysteries, with properties that do not fit cleanly into our current models of radio galaxies. It is not clear whether this is even a standard twin-tailed source, with the tails merged in projection or physically, or whether it is new type of one-sided structure. While a variety of factors could be responsible for the bright 
patches down the tail and the accompanying "ribs", none of them satisfactorily accounts for all the observed properties. Added to this puzzle, the continuity of structure, spectrum, and rotation measures from the far tail, through the tethers, to the triple source, suggests that they are one structure, but the apparent host at a different redshift must then be viewed as an improbable coincidence. If we are indeed looking at one structure, then the tethers and perhaps the ribs, likely belong to the newly emerging examples of thin magnetized threads linking larger regions of relativistic plasma-i.e., an exciting new physical phenomenon.

Author Contributions: Conceptualization, L.R.; data curation, W.C.; writing-original draft preparation, L.R.; writing - review and editing, K.K. (Kenda Knowles) and K.K. (Konstantinos Kolokythas). All authors have read and agreed to the published version of the manuscript.

Funding: L.R.'s effort was funded in part by U.S. National Science Foundation grant AST17-14205 to the University of Minnesota. MGCLS data products were provided by the South African Radio Astronomy Observatory and the MGCLS team and were derived from observations with the MeerKAT radio telescope. The MeerKAT telescope is operated by the South African Radio Astronomy Observatory, which is a facility of the National Research Foundation, an agency of the Department of Science and Innovation. K.Kn. is supported by the New Scientific Frontiers grant of the South African Radio Astronomy Observatory.

Acknowledgments: We thank T.W. Jones, C. Nolting, C. Pfrommer, B. McKinley, and Gopal-Krishna for interesting ideas on the origins of the features observed here and H. Akamatsu for providing the XMM image.

Conflicts of Interest: The authors declare no conflict of interest.

\section{References}

1. Knowles, K.; Cotton, W.; Rudnick, L.; Camilo, F.; Goedhart, S.; Deane, R.; Ramatsoku, M.; Bietenholz, M.; Brüggen, M.; Button, C.; et al. The MeerKAT Galaxy Cluster Legacy Survey. Astron. Astrophys. submitted.

2. Dehghan, S.; Johnston-Hollitt, M.; Colless, M.; Miller, R. Dynamics of Abell 3266-I. An optical view of a complex merging cluster. Mon. Not. R. Astron. Soc. 2017, 468, 2645-2654. [CrossRef]

3. Bernardi, G.; Venturi, T.; Cassano, R.; Dallacasa, D.; Brunetti, G.; Cuciti, V.; Johnston-Hollitt, M.; Oozeer, N.; Parekh, V.; Smirnov, O.M. KAT-7 observations of an unbiased sample of mass-selected galaxy clusters. Mon. Not. R. Astron. Soc. 2016, 456, 1259-1268. [CrossRef]

4. Finoguenov, A.; Henriksen, M.J.; Miniati, F.; Briel, U.G.; Jones, C. A Puzzling Merger in A3266: The Hydrodynamic Picture from XMM-Newton. Astrophys. J. 2006, 643, 790. [CrossRef]

5. Sanders, J.S.; Biffi, V.; Brüggen, M.; Bulbul, E.; Dennerl, K.; Dolag, K.; Erben, T.; Freyberg, M.; Gatuzz, E.; Ghirardini, V.; et al. Studying the merging cluster Abell 3266 with eROSITA. arXiv 2021, arXiv:2106.14534.

6. Zou, H.; Gao, J.; Zhou, X.; Kong, X. Photometric Redshifts and Stellar Masses for Galaxies from the DESI Legacy Imaging Surveys. Astrophys. J. Suppl. Ser. 2019, 242, 8. [CrossRef]

7. Katz-Stone, D.M.; Rudnick, L. An Analysis of the Synchrotron Spectrum in the Fanaroff-Riley Type I Galaxy 3C 449. Astrophys. J. 1997 486, 146. [CrossRef]

8. Godfrey, L.E.H.; Lovell, J.E.J.; Burke-Spolaor, S.; Ekers, R.; Bicknell, G.V.; Birkinshaw, M.; Worrall, D.M.; Jauncey, D.L.; Schwartz, D.A.; Marshall, H.L.; et al. Periodic Structure in the Megaparsec-scale Jet of PKS 0637-752. Astrophys. J. 2012, 758, L27. [CrossRef]

9. Lister, M.L.; Homan, D.C.; Kellermann, K.I.; Kovalev, Y.Y.; Pushkarev, A.B.; Ros, E.; Savolainen, T. MOJAVE: XVIII. Kinematics and Inner Jet Evolution of Bright Radio-Loud Active Galaxies. arXiv 2021, arXiv:2108.13358.

10. Hota, A.; Sirothia, S.K.; Ohyama, Y.; Konar, C.; Kim, S.; Rey, S.; Saikia, D.J.; Croston, J.H.; Matsushita, S. Discovery of a spiral-host episodic radio galaxy. Mon. Not. R. Astron. Soc. 2011, 417, L36-L40. [CrossRef]

11. Birzan, L.; Rafferty, D.A.; Nulsen, P.E.J.; McNamara, B.R.; Röttgering, H.J.A.; Wise, M.W. The duty cycle of the radio mode feedback. Astron. Nachr. 2013, 334, 390-393. [CrossRef]

12. Barniol Duran, R.; Tchekhovskoy, A.; Giannios, D. Simulations of AGN jets: Magnetic kink instability versus conical shocks. Mon. Not. R. Astron. Soc. 2017,469, 4957-4978. [CrossRef]

13. O'Neill, B.J.; Jones, T.W.; Nolting, C.; Mendygral, P.J. A Fresh Look at Narrow-angle Tail Radio Galaxy Dynamics, Evolution, and Emissions. Astrophys. J. 2019, 884, 12. [CrossRef]

14. Nolting, C.; Jones, T.W.; O'Neill, B.J.; Mendygral, P. Interactions between Radio Galaxies and Cluster Shocks. I. Jet Axes Aligned with Shock Normals. Astrophys. J. 2019, 876, 154. [CrossRef]

15. Sanders, R.H. The reconfinement of jets. Astrophys. J. 1983, 266, 73. [CrossRef]

16. Mizuno, Y.; Gómez, J.L.; Nishikawa, K.I.; Meli, A.; Hardee, P.E.; Rezzolla, L. Recollimation Shocks in Magnetized Relativistic Jets. Astrophys. J. 2015, 809, 38. [CrossRef]

17. McKinley, B.; Tingay, S.J.; Gaspari, M. Multi-scale feedback and feeding in the closest radio galaxy Centaurus A. submitted. 
18. van Weeren, R.J.; Shimwell, T.W.; Botteon, A.; Brunetti, G.; Brüggen, M.; Boxelaar, J.M.; Cassano, R.; Gennaro, G.D.; AndradeSantos, F.; Bonnassieux, E.; et al. LOFAR observations of galaxy clusters in HETDEX. Extraction and self-calibration of individual LOFAR targets. Astron. Astrophys. 2021, 651, 115. [CrossRef]

19. Ramatsoku, M.; Murgia, M.; Vacca, V.; Serra, P.; Makhathini, S.; Govoni, F.; Smirnov, O.; Andati, L.A.L.; de Blok, E.; Józsa, G.I.G.; et al. Collimated synchrotron threads linking the radio lobes of ESO 137-006. Astron. Astrophys. 2020, 636, L1. [CrossRef]

20. Condon, J.; Cotton, W.; White, S.; Legodi, S.; Goedhart, S.; McAlpine, K.; Ratcliffe, S.M.; Camilo, F. Threads, Ribbons, and Rings in the Radio Galaxy IC 4296. ApJ 2021, 917, 18. [CrossRef]

21. Lane, W.M.; Kassim, N.E.; Ensslin, T.A.; Harris, D.E.; Perley, R.A. 3C 129 at 90 Centimeters: Evidence for a Radio Relic? Astron. J. 2002, 123, 2985. [CrossRef]

22. Hardcastle, M.J.; Croston, J.H.; Shimwell, T.W.; Tasse, C.; Gürkan, G.; Morganti, R.; Murgia, M.; Röttgering, H.J.A.; van Weeren, R.J.; Williams, W.L. NGC 326: X-shaped no more. Mon. Not. R. Astron. Soc. 2019, 488, 3416-3422. [CrossRef]

23. Thomas, T.; Pfrommer, C.; Enßlin, T. Probing Cosmic Ray Transport with Radio Synchrotron Harps in the Galactic Center. Astrophys. J. Lett. 2020, 890, L18. [CrossRef]

24. Jones, A.; de Gasperin, F.; Cuciti, V.; Hoang, D.N.; Botteon, A.; Brüggen, M.; Brunetti, G.; Finner, K.; Forman, W.R.; Jones, C.; et al. Radio relics in PSZ2 G096.88+24.18: A connection with pre-existing plasma. Mon. Not. R. Astron. Soc. 2021, 505, 4762. [CrossRef] 